\title{
Analysis of Turbulent Axisymmetric Near Wake
}

\author{
T. Yane and N. Subaschandar
}

Department.of Mathematics and Computational Sciences, BIUST, P.B.16, Palapye, Botswana

\begin{abstract}
Axisymmetric near wake flow behind a body of revolution has been analysed. It is shown that the logarithmic layer of the upstream turbulent boundary layer continues to be valid for some more distance in the near wake and as the streamwise distance is increased the logarithmic layer is slowly getting destroyed. It is also shown that the central line velocity exhibits a logarithmic behaviour with streamwise distance.
\end{abstract}

\section{Introduction}

Turbulent flows are ubiquitous in natural phenomena and engineering applications [1] therefore a mathematically tractable description of them is desirable for their prediction and control. Flow region near an afterbody of an airborne vehicle has technological as well as scientific interest on account of its contribution to aerodynamic drag. The turbulent boundary layer approaching the base is subjected to large pressure gradient and to longitudinal and transverse curvature. It converts vorticity into the recirculating region near the base. On the other hand, the latter has an upstream influence on the boundary layer. This interaction is weak in supersonic case and is relatively well understood, but the problem is more serious in subsonic flow cases.

The near wake is the region of the wake flow just behind the trailing edge of the body where flow development takes place without the constraints imposed by the body. Turbulent wakes are one of the least studied flows, mostly due to their long development length and the difficulty in measuring low-level turbulent intensities in the far wake. Most of the previous works have focused on the behaviour of planewakes, such as those produced by circular cylinders, flat plates and airfoils[2-5]. Most of the analytical work available in the literature are related to two-dimensional turbulent near wake flows[6-8]. Alber[6] studied the development of the turbulent near wake of a thin flat plate using method of asymptotic expansions. His analysis showed that the velocity profile varied logarithmically with normal distance in the inner near wake. Subaschandar and Prahbu[7] carried out an asymptotic analysis of a two-dimensional turbulent near wake behind a flat plate. Walker et al[8] provided an analytical solution to the two-dimensional turbulent near wake problem. These reports indicated that a logarithmic variation in the normal direction exists in the overlapping region between the inner near wake and the outer near wake regions.

The axisymmetric turbulent wake is a flow that has puzzled researchers for more than a half-century, since measured results have been either inconclusive or contradictive. The axisymmetric wake is a challenging flow to measure because of the small velocity deficit, the slow decay of the velocity deficit downstream and a turbulence intensity of the same order as the deficit. In fact, the far axisymmetric wake still is at the threshold of what is possible to measure today using even the best wind tunnels and the most stable low-noise anemometer equipment. Also, unlike many other free shear flows for which the local Reynolds number remains constant or increases downstream, in the axisymmetric wake it drops slowly. Thus viscous effects continuously become more important until eventually they may dominate (if the flow extends far enough downstream). These varying viscous effects, together with the many very different possibilities for the structure of the near wake from different generators, considerably complicates interpretation of the data. Axisymmetric turbulent near wake flow has not been studied very well. Available research reports on axisymmetric near wake are pertaining to experimental works[9-13]. These studies essentially highlight special features of thick axisymmetric turbulent boundary layers and axisymmetric turbulent near wake regions. There are no analytical works that describe the axisymmetric inner near wake behaviour.

The present work makes an attempt to describe the behaviour of mean velocity profiles in the inner near wake behind behind a long slender cylinder. The turbulent flows, because of memory, take long time and distance before adjusting and coming to equilibrium with a new environment[14]. In view of this memory, it is reasonable to assume the velocity and length scales determining the turbulent boundary layer near training 
edge should also be relevant velocity and length scales for the near-wake.

\section{Model for near wake:}

Figure 1 gives the schematic of the problem along with the coordinate system and the corresponding velocity components. In this study, we focus our attention in the inner near wake region. The governing equations for inner near wake can be obtained by making a local asymptotic analysis, similar to that made for a fully developed turbulent boundary layer and two-dimensional turbulent near wake[7,15]. The limiting conditions are $\left(\frac{\mathrm{U}_{\tau}}{\mathrm{U}_{\mathrm{\tau}}}\right) \rightarrow 0$ and $\left(\frac{\mathrm{BU}_{\tau}}{\mathrm{v}}\right) \rightarrow \infty$, where $\mathrm{U}_{\tau}$ is the skin friction velocity at the trailing edge. Under these limiting conditions the non-dimensionalised governing equations for inner and outer near wake regions are

$$
\begin{gathered}
\frac{\partial\left(\mathrm{y}_{+} \mathrm{U}_{+}\right)}{\partial \mathrm{x}_{+}}+\frac{\partial\left(\mathrm{y}_{+} \mathrm{v}_{+}\right)}{\partial \mathrm{y}_{+}}=0 \\
\mathrm{U}_{+} \frac{\partial \mathrm{U}_{+}}{\partial \mathrm{x}_{+}}+\mathrm{V}_{+} \frac{\partial \mathrm{U}_{+}}{\partial \mathrm{y}_{+}}+\frac{1}{\mathrm{y}_{+}} \frac{\partial}{\partial \mathrm{y}_{+}}\left\{\mathrm{ky_{+ }} \frac{\partial \mathrm{U}_{+}}{\partial \mathrm{y}_{+}}\right\}=0
\end{gathered}
$$

where $\mathrm{U}_{+}=\frac{\mathrm{U}}{\mathrm{U}_{\tau}}, \mathrm{V}_{+}=\frac{\mathrm{v}}{\mathrm{U}_{\tau}}, \mathrm{y}_{+}=\frac{\mathrm{YU}_{\tau}}{\mathrm{v}}, \mathrm{x}_{+}=$

$$
\frac{\mathrm{xu}_{\tau}}{\mathrm{v}}, U_{d}=\frac{U_{\infty}-U}{U_{\tau}}, \tau=\mu_{\tau} \frac{\partial U_{+}}{\partial y_{+}} \text {. }
$$

For simplicity of the analysis, an assumption, similar to the one made in reference[6], for the eddy viscosity is given by $\boldsymbol{\mu}_{\mathbf{r}}=\mathbf{k U}_{\mathbf{\tau}} \mathbf{y}_{+}$. The matching between the inner and outer near wake leads to the boundary conditions in the overlapping region as $\frac{\mathbf{v}}{\mathbf{U}_{\mathbf{z}}}=\frac{1}{\mathbf{k}} \ln \frac{\mathrm{y} \mathbf{U}_{\mathbf{v}}}{\mathbf{v}}+\mathbf{B}_{\mathbf{1}}$, where $B_{\mathbf{1}}$ is a constant. $B_{1}$ depends on the radius of the cylindrical body[16].

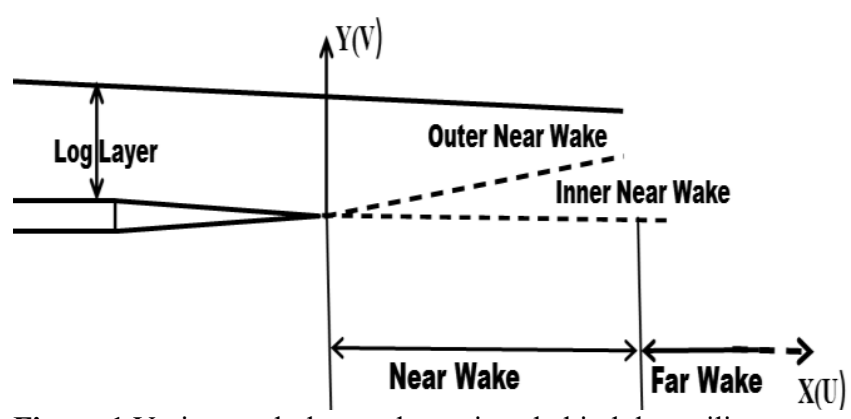

Figure 1 Various turbulent wake regions behind the trailing edge of an axisymmetric body

\section{Analysis}

We seek for a solution of Eq. 2 and the continuity Eq. 1 which is valid for a large $y_{+}$such that for a fixed value of $\mathrm{X}_{+}$,

$$
\lim _{y_{+} \rightarrow \infty} U_{+} \sim \frac{1}{k} \ln y_{+}+B_{1} .
$$

This suggests a solution of the form,

$$
\mathrm{U}_{+}=\mathrm{S}\left(\mathrm{X}_{+}\right)+\frac{1}{\mathrm{k}} \mathrm{F}^{\prime}(\eta)
$$

Where $\eta=\frac{Y_{+}}{g\left(X_{+}\right)}$and prime denotes differentiation with respect to $\eta$ or $X_{+}$. We can get an equation in terms of $\boldsymbol{V}_{+}$from the continuity Eq. 1 and substituting into the momentum Eq. 2 to get an equation as follows;

$$
\begin{aligned}
& \frac{1}{\eta}\left[\eta^{2} F^{\prime \prime}\right]^{\prime}-\frac{g s^{\prime}}{\mathrm{k}}\left[\mathrm{F}^{\prime \prime} \eta\right]+g S S^{\prime}=\frac{\mathrm{gs}}{\mathrm{k}}\left[\frac{\eta \mathrm{F}^{\prime \prime}}{2}-\right. \\
& \left.\mathrm{F}^{\prime}\right]+\frac{2 \mathrm{~g} \mathrm{~g}^{\prime}}{\mathrm{k}^{2}}\left[\mathrm{FF}^{\prime \prime}-\right. \\
& \left.\frac{\mathrm{F}}{\eta} \int_{0}^{\eta} \mathrm{Fd} \eta\right] .
\end{aligned}
$$

To obtain the similarity equation for $\bar{F}$ we set the two functions of $\mathrm{X}_{+}$appearing in the equation to constants. Without loss of generality we may set the constants to 1 , that is,

$$
\frac{\mathrm{gS} i}{\mathrm{k}}=1 \text { and } \mathrm{gSS}^{\prime}=1
$$

On solving to get equations of $S$ and $g$ yields,

$$
S\left(X_{+}\right)=\frac{1}{k} \ln (g) \text { and } g[\ln (g)-1]=k^{2} X_{+}
$$

Using these equations in Eq. 5, we get

$$
\begin{aligned}
& \frac{1}{\eta}\left[\eta^{2} \mathrm{~F}^{\prime \prime}\right]^{\prime}-\left[\mathrm{F}^{\prime \prime} \eta\right]+1=\frac{1}{\ln \mathrm{g}}\left[\eta \frac{\mathrm{F}^{\prime \prime}}{2}-\mathrm{F}^{\prime}+\right. \\
& \mathrm{FF}^{\prime \prime}- \\
& \left.\frac{\mathrm{F}^{\prime \prime}}{\eta} \int_{0}^{\eta} \mathrm{Fd} \eta\right] .
\end{aligned}
$$

For a relatively large $\ln (g)$, we get

$$
\left[\eta^{2} F^{\prime \prime}\right]^{\prime}-\left[\eta^{2} F^{\prime \prime}\right]+\eta=0 .
$$

The boundary conditions for $F(\eta)$ at the axis are given by the requirement that $V_{+}=0$ on $y_{+}=0$ and hence the centreline conditions are;

$$
F^{\prime}(0)=0 \text { and } \lim _{\eta \rightarrow \infty} \eta F^{\prime \prime}=0 .
$$

To satisfy the boundary conditions imposed by Eq. 3 for a large $\mathrm{Y}_{+}$at a fixed $\mathrm{X}_{+}$we require that 


$$
\lim _{\eta \rightarrow \infty} F^{\prime} \rightarrow \ln \eta+B k
$$

\section{Similarity solution for $\boldsymbol{F}_{\mathbf{0}}^{\prime}$}

The solution to the differential Eq. 9 subject to the previously stated boundary condition is,

$$
\mathrm{F}^{\prime \prime}=\frac{\eta-1}{\eta^{2}}
$$

Integrating to obtain a solution for $F^{\prime}$ yields,

$$
\mathrm{F}^{\prime}=\ln \eta+\frac{1}{\eta}
$$

Using the asymptotic boundary conditions given by Eq. 11, Eq. 13 reduces to

$$
\lim _{\eta \rightarrow \infty} F^{\prime}(\eta) \sim F^{\prime}(0)+B_{1}+\frac{1}{\eta}+\ln \eta \sim \ln \eta+B k .
$$

and hence,

$$
\mathrm{F}^{\prime}(\mathrm{O})=\mathrm{Bk}-\mathrm{B}_{1} \text {. }
$$

Rewriting Eq. 14 using Eq.15 we have;

$$
\mathrm{F}^{\prime}=\ln \eta+\varphi(\eta)+\mathrm{Bk}-\mathrm{B}_{1}
$$

\section{Experimetal data for comparison}

Patel and Lee [8] carried out experimental studies in the axisymmetric near wake behind a body of revolution. Reynolds number in their experiments, based on the body length and freestream velocity was $1.2 \times 10^{\mathfrak{B}}$. On analysing the pressure distribution data given in report[8], it was observed that the pressure gradients in the freestream and normal direction were small and hence their dataset was considered for comparing the analytical results of the present study. Jimeniz at al [9] performed measurements in the axisymmetric wake of a long slender cylinder and the Reynolds number range was $1.6 \times 10^{\mathrm{B}}$ to $67 \times 10^{\mathrm{G}}$. The results of the present analysis were compared with the experimental data of Jimenez at al [9], but not presented here because of page limit.

\section{Results and discussion}

Figure 2 presents the variation of mean velocity profiles in the normal direction at different streamwise stations, comparing with the experimental data[8]. This figure shows the logarithmic lines and shows the existence of logarithmic layer in the inner near wake. The logarithmic layer slowly gets destroyed as the streamwise distance increases.

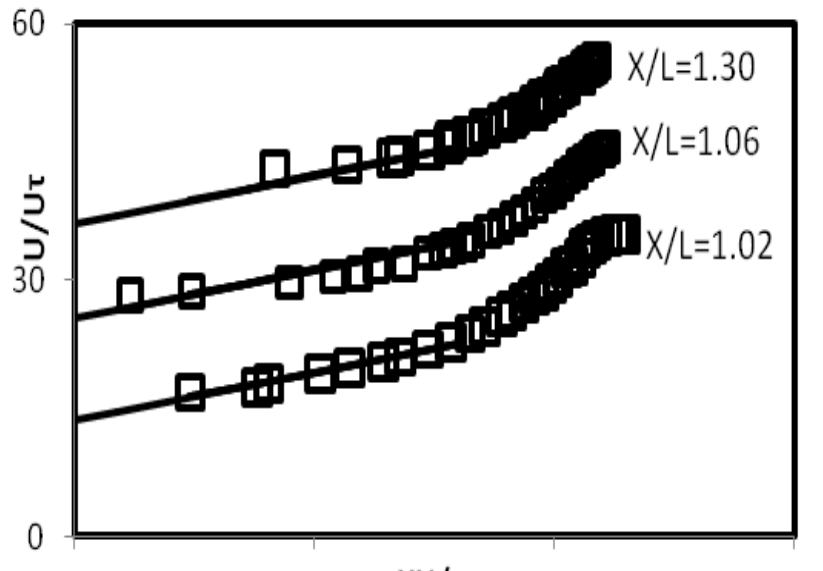

$\begin{array}{lll}10 & \mathrm{YU} / \mathrm{V} \quad 10000\end{array}$ Figure 2 Logarithmic variation of streamwise velocity in the
normal direction. $\square$ experimental data[8], log line.

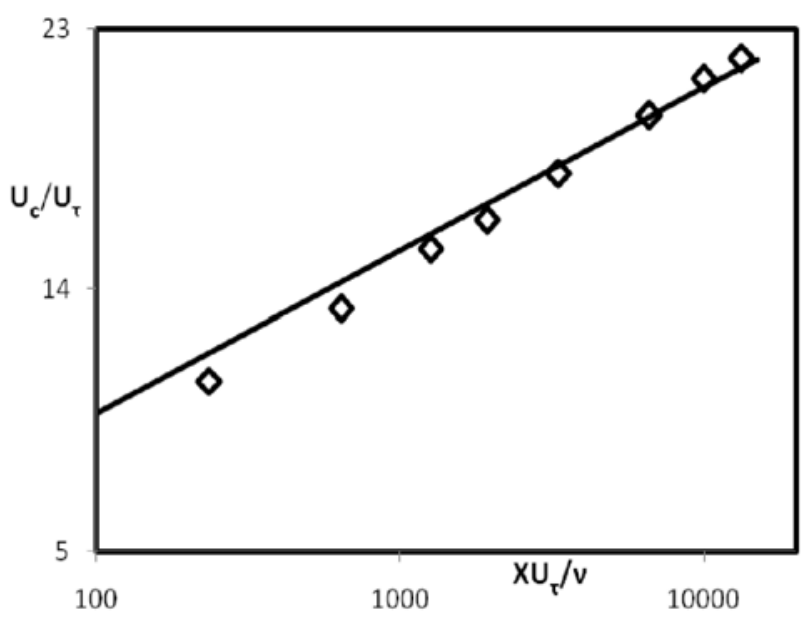

Figure 3 Variation of the centerline velocity with streamwise distance. $\diamond$ Experimental data[8], $\longrightarrow$ log line.

The behaviour of the solution for large $\mathrm{X}_{+}$in the inner near wake can be seen from the variations of the centerline velocity given by $\mathrm{S}\left(\mathrm{X}_{+}\right)$and the length scale of the inner wake given by $\mathrm{g}$ from the Eq. 7. Taking logarithm on both sides of the second equation of Eq. 7 the relation between $g$ and $X_{+}$becomes more explicit in the form

$\ln (g)+\ln [\ln (g)-1]=2 \ln \mathrm{k}+\ln \left(\mathrm{X}_{+}\right)$.

This shows that, to the first order, $\ln (\mathrm{g})$ varies like $\ln \left(\mathrm{X}_{+}\right)$. Substituting for $\ln (\mathrm{g})$ in the Eqs. 7 , we get a relation for $\mathrm{S}\left(\mathrm{X}_{+}\right)$as

$$
s\left(\mathrm{X}_{+}\right)=\frac{1}{\mathrm{k}}\left[\ln \left(\mathrm{X}_{+}\right)-\ln [\ln (\mathrm{g})-1]+2 \operatorname{lnk}+\mathrm{C} .\right.
$$

This shows that the centerline velocity variation for large $X_{+}$is dominated by the term $(1 / k) \operatorname{In}\left(X_{+}\right)$. The other terms are very much small compared to this term. The variation of $\operatorname{In}(\mathrm{g})$, can also be considered small over the region of interest in $\mathrm{X}_{+}$, which is of couple of decades' extent, and for all practical purposes $\mathrm{S}$ can be considered to vary like 


$$
\mathrm{S}=\frac{1}{\mathrm{k}} \ln \left(\left(\mathrm{x}_{+}\right)+\mathrm{C}_{1}\right.
$$

over the range of interest in the downstream direction for large $\mathrm{X}_{+} \mathrm{C}_{1}$ is a constant which can be evaluated from experimental data. Figure 3 shows the variation of the centreline velocity with streamwise distance for the experimental data[8]. Figure 3 shows the logarithmic line and we can see the logarithmic variation of the centreline velocity for large streamwise distance, as given by Eq. 19 . Logarithic behaviour of mean velocity profiles has been reported in the experimental data given in[12] also.

In summary, mean velocity profiles, in the inner near wake, vary logarithmically with distance in the normal as well as streamwise directions.

\section{Conclusions}

An axisymmetric turbulent inner near wake development has been studied analytically. It is shown that the same limiting condition $\frac{\mathbf{U}_{\mathbf{x}}}{\mathbf{U}_{\mathrm{w}}} \rightarrow \mathbf{0}$ as used for an asymptotic treatment of a fully developed axisymmetric turbulent boundary layer for a large Reynolds number is applicable to the analysis of the axisymmetric turbulent near wake. The streamwise component of the mean velocity has been shown to vary logarithmically with normal distance in the normal direction in the inner near wake. This logarithmic layer is getting destroyed slowly as the streamwise distance increases. The centreline velocity is shown to vary logarithmically in the streamwise direction for large streamwise distances. Available experimental data validate the results of the analysis.

\section{Acknowledgements}

Authors acknowledge the support received from the Department of Mathematics and computational Sciences and BIUST authorities in carrying out this research.

\section{References}

1. S. B. Pope, Turbulent flows, Cambridge University Press (2000).

2. S. J. Goldstein, J. Aeronaut. Sci. 15, 189 (1948).

3. A. A. Townsend, The Structure of Turbulent Shear Flow. Cambridge University Press. (1956).

4. I. Wygnanski, F. Champagne, \& B. Marasli, J. Fluid Mech. 168, 31 (1986).

5. H. Oertel, Annual Rev. Fluid Mech. 22, 539 (1990).

6. I. E. Alber, AIAA J., 18, 1044 (1980).

7. N. Subaschandar and A. Prabhu, J. Aerospace Science and Technology., 1, 1089 (1999)

8. J. D. A.Walker and D. Bocucz, J. of Fluid Mech., 112, 100 (1988).

9. V. C. Patel and Y. T. Lee, IIHR Report 210, IIHR, The Univ. of Iowa, USA. (1977).

10. J. M. Jimenez, M. Hultmark, and A. J. Smits, J. of Fluid Mech., 659, 516 (2010).
11. V. C. Patel, Y. T. Lee, and O. Guven, In Turbulent Shear Flows - I , Springer-Verlag, Berlin, 137 (1979).

12. R. A. Merz, R. H. Page, and C. E. G. Prziregel, AIAA J., 16, 656 (1978).

13. V. Krishnan and K. S. Yajnik, Proceedings of $10^{\text {th }}$ Australasian Fluid Mech. Conf., University of Melbourne, DEC 1989, 9.26 (1989).

14. R. Narasimha and A. Prabhu, J. of Fluid Mech., 54, 1 (1972)

15. N. Afzal and R. Narasimha, J. of Fluid Mech., 74, 113 (1976).

16. G. N. V. Rao and N. R. Keshavan, J. of Applied Mech., 39, 1, 25 (1972). 